Willis. For a clue of resolving this unknown etiology we reported a case which showed coexistence of glioma of the optic chiasm and the peculiar vascular network.

\title{
D-9. Clinical Experience on "Intracranial Peculiar Vascularity with Localized Stenosis of Distal End of Internal Carotid Arteries"
}

\author{
Kinjiro Iwata \\ Department of Surgery, Nagoya University Hospital
}

Thirteen cases of today's topic disease are presented at this symposium. They were personally observed and followed-up in the past eight years at the Nagoya University Hospital. Eight cases out of the thirteen were considered typical because they were common in the clinical picture of repeated episodes of sudden onset, and short period hemiplegia which disappeared in 10 days or so. Headache accompanies most cases at the onset but not as severe as seen in subarachnoid hemorrhage. Laterality of hemiplegia is altered in some cases and aphasic disturbances usually is accompanied with right-side motor distrurbance. Lumbar puncture at the acute stage revealed no evidence of subarachnoid bleeding in my series, but it usually demonstrated moderate degree of increased protein and mild pleocytosis in the cerebral spinal fluid. EEG is interpretated as non-epileptogenic in pattern in most cases.

Angiographic study revealed localized stenosis or obstruction of the distal end of internal carotid arteries or absence of their major branches, such as the the middle cerebral arteries. Ophthalmic arteries and posterior communication arteries were usually spared from vascular stenotic changes. In addition to those stenotic picture, peculiar vascularity is seen in the center of the brain, almost entirely in the region of the diencephalon and centrum semiovale of the cerebral hemisphere. On angiographic studies, including serial angiogram, this peculiar vascularity seems to be essentially arterial in nature.

Vertebral artery system is not involved, except it often supples blood circulation into this peculiar vascular pattern through posterior cerebral circulation. These changes were seen bilaterally in my series and symmetrically to a more or less extent.

Five cases out of my thirteen are categorized atypical for this abnormality because they showed some pattern of arterio-venous anastomosis demonstrated on angiogram, but otherwise, the change was seen to have essentially the same pattern. Atypical cases were almost always in adult patients, and clinical picture 
varied from attacks of headaches, convulsive fits to repeated episodes of amaurosis.

To discuss the nature, especially the etiology of this peculiar cerebral vascular disease, the author has the opinion that this peculiar vascularity in the deep structure of the brain controls the function of blood supply for brain metabolism to compensate for poor circulation due to stenosis or obstruction of bilateral internal carotid arteries or their major branches. This seems collateral in nature from the angiographical and clinical standpoints.

We have had no cases of death and thus no histological examination have been made. The histological study on those abnormal vessels and the stenotic carotid wall should give some clue to determine the etiology of this disorder.

\title{
D-10. Cerebral Basal Rete Mirabile
}

\author{
Akira Nishimoto and Shinji TAKeUchr \\ Dept. of Neurological Surgery, Okayama University Medical School
}

The authors presented statistics of total 96 cases of this disease including the authors' own cases at the 24th Annual Meeting of Japan Neurosurgical Society, which were collected from literature and by letter inquiry into neurosurgical clinics in Japan 1965, and suggested that there is a marked difference in its clinical symptoms between children and adults. Moreover, the authors emphasized that serial bilateral carotid and vertebral angiography should be done to distinguish those cases from other occlusive diseases of internal cortid artery. Up to the present, 9 cases of this disease have been experienced in the authors' clinic.

This time, the authors present our own opinion about the cause of this disease, which is thought to be congenital malformation. The reasons of congenital malformation theory are; 1) Typical angiographic findings are usually seen bilaterally as well as symmetrically. 2) Stenosis or occlusion and abnormal vascular network are seen only in the area of the internal carotid artery, while external carotid artery and vertebro-basilar arteries are quite normal. Therefore, the authors used the term "Hemangiomatous Malformation of Bilateral Internal Carotid Artery at the Base of Brain". 3) Internal carotid artery is usually thin and narrow bilaterally in its total course from the bifurcation to the site of occlusion. Anterior and middle cerebral arteries, even if visualized, are usually of poor and irregular filling, and they do not originate directly from internal carotid, but from the vascular network. Thus, the internal carotid artery demonstrates an appearance of congenital malformation from the bifurcation to the peripheral area. 4) If an acute stenosis or occlusion occur at the site of carotid siphon, it must cause severe neurological symptoms even by unilateral one. In this cerebral basal rete mirabile disease, how- 